\title{
Preface
}

\section{Thoracic Surgery in the Special Care Patient}

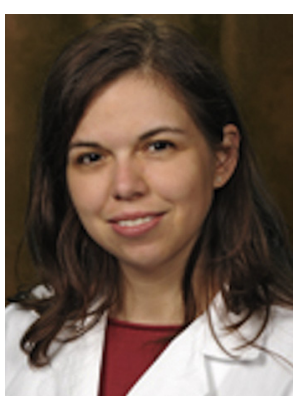

Sharon Ben-Or, MD Editor

Every thoracic surgeon has encountered a patient whose problem is outside of the box, a clinical conundrum. Often, the diagnosis and workup can be as vexing as formulating a treatment plan. These patients require thoughtful and methodical care often with the input of multiple physicians from multiple subspecialties. Evidence for these disease processes exists, but guidelines are scarce. From the pregnant patient to the lung transplant recipient with lung cancer to the patient who underwent weight reduction surgery with esophageal cancer, these unique patients require the attention and innovation of a thoracic surgeon who sometimes has to push the envelope in order to treat the patient.

This issue of Thoracic Surgery Clinics focuses on the complex diagnoses of these special care patients that do not fit any particular category.
These cases are rarely encountered but are always memorable. I would like to thank each contributor for taking the time to reflect on their own past experiences and pitfalls of each of these patients. As we pull back the curtain on these rare clinical challenges, we hope to shed light on how to appropriately treat these unforgettable patients.
Sharon Ben-Or, MD Division of Thoracic Surgery Department of Surgery Greenville Memorial Hospital 890 West Faris Road, Suite 320 Greenville, SC 29605, USA

E-mail address: SBen-Or@ghs.org 